\title{
Data Sharing Model for Sequence Alignment to Reduce Database Retrieve*
}

\author{
Min Jun Kim ${ }^{1}$, Jai-Hoon Kim ${ }^{1}$, Jin-Won Jung ${ }^{2}$, and Weontae Lee ${ }^{2}$ \\ ${ }^{1}$ The Graduate School of Information and Communication, Ajou University \\ Suwon, 442-749, Republic of Korea \\ \{xwind, jaikim\}@ajou.ac.kr \\ ${ }^{2}$ HTSD-NMR Laboratory, Department of Biochemistry \\ Yonsei University, Seoul 120-749, Republic of Korea \\ \{solwind, wlee\} @spin.yonsei.ac.kr
}

\begin{abstract}
Many programs of bioinformatics provide biochemists and biologists retrieve and analysis services of gene and protein databases. These services access databases for each arrival of user's request, which takes a long time, increases server's overload and response time. In this paper, two data sharing models are presented to reduce database retrieve for sequence alignments. (1) Grouping model is proposed to share database access between many requests arrived in a certain period of time by utilizing database access patterns in bioinformatics. (2) Carpool model is also proposed to reduce response time as well as to increase system throughput by servicing new arriving request immediately together with the previous on going requests to share database accesses without waiting time. Simulation results show that two data sharing models can reduce the number of database access by sharing it among many requests and increase system throughput.
\end{abstract}

\section{Introduction}

In the early $21^{\text {st }}$ century, success in human gene project accelerates development in bio-science technology. In the Postgenome era spread by completion of the human genetic map, researches about structures and functions of proteins and discovery of genes will be performed a lot. These researches produce huge quantity of gene data. Gene data is expressed by four characters (A, T, G, C). Data more than three billion DNA bases are accumulated in bio-database, many of which can be accessed via Web. There are many popular bio-databases such as SwissProt [6], GenBank [7], EMBL [8] etc.

Whole records in database are retrieved in many bioinformatics applications such as sequence alignments and structure comparisons. The order of data retrieve does not affect on the results in many applications. We propose new data sharing models to

\footnotetext{
* This study was supported by the Brain Korea 21 Project in 2003, a grant of the International Mobile Telecommunications 2000 R\&D Project, Ministry of Information \& Communication, Republic of Korea, and the Ministry of Science and Technology of Korea / the Korea Science and Engineering Foundation through the NRL program of MOST NRDP (M1-0203-00-0020).
} 
increase performance of applications that access bio-databases in this characteristic. First, grouping model is proposed, which accesses database records once and process several requests together by gathering many users' requests arrived in a scheduled period. Grouping model can decrease the number of database access and reduce total system cost. Second, carpool model is proposed to avoid waiting time until the end of period for grouping users' requests unlike the grouping model. New arrival of request is combined with the previous on going requests at any time and shares the database accesses in the carpool model, while new request has to wait until the end of grouping period in the grouping model. Carpool model can reduce an average response time for each user as well as the number of total database accesses.

\section{Traditional Model}

Many applications of bioinformatics perform gene sequence alignments and protein structure comparisons between genes and proteins. These applications and gene and protein databases are provided on the Web. In such an environment, users send gene or protein sequences through the Web. Then, programs on server such as FastA and Blast read known sequences in databases and compare them with sequences contained in users' requests.

\subsection{Traditional Architecture}

Many programs used for sequence alignments or structure comparisons retrieve the whole records in gene databases such as SwissProt [6], GenBank [7], EMBL [8], etc. Such programs as FastA and Blast must respond to each user's request after retrieving whole records in database and comparing each of records with user's sequence.

In traditional model, new arrival of request is immediately processed without waiting time when system is idle. However, when the previous request is processing, new request is registered to a queue in the order of arrival, then processed only after all the previous requests are serviced.

\subsection{Cost Analysis}

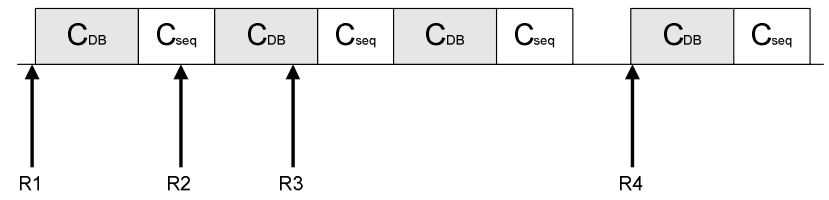

Fig. 1. Traditional program model

Figure 1 shows the timing diagram to demonstrate arrival and servicing the users' requests, R1 to R4. $C_{D B}$ denotes an assumed cost for database retrieve for all re- 
cords. Also, $C_{s e q}$ denotes an assumed cost for comparison between gene sequence from a user request and all gene sequences from a database.

An average cost to process a user request for a gene sequence alignment is the sum of database retrieve cost $\left(C_{D B}\right)$ and comparison cost $\left(C_{s e q}\right)$ between gene sequences. Thus, the average cost for a request, $C_{\text {avg }}^{\text {org }}$, can be computed as follows:

$$
C_{\text {avg }}^{\text {org }}=C_{D B}+C_{s e q}
$$

It is assumed that the arrival of user request is Poisson process with an arrival rate $\lambda$. User's request is registered to a queue by the order of arrival, and processed sequentially. It is M/G/1 queuing model. The service rate is $1 / C_{\text {arg }}^{\text {org }}$. Using M/G/1 queuing model, response time can be derived as follows:

$$
w_{o}=C_{\text {avg }}^{\text {org }}+\frac{\lambda C_{\text {avg }}^{\text {org } 2}}{2\left(1-\lambda \cdot C_{\text {avg }}^{\text {org }}\right)}
$$

\section{Grouping Model}

Problem in the traditional model is retrieving the whole records in a database for a sequence alignment on each arrival of user's request. Bioinformatics programs like FastA and Blast have to access whole records in the database repeatedly to service many users' requests. Grouping model reduces the number of database access by grouping users' requests and sharing the database access. Thus, it can reduce a total system cost and increase system throughput.

\subsection{Grouping Architecture}

Grouping model gathers users' requests and processes together periodically without accessing a database every time on each user's request. Grouping model retrieves sequences from a database once in a period, it compares between gene sequences in the database and gathered sequences from users' requests in a period. By doing so, grouping model can reduce the number of database access remarkably because it retrieves records in the database once in a period without accessing database individually for each request.

In Fig. 2, Rj denotes j-th users' requests arrived in the i-th period. Users' requests are processed after the end of i-th period. For grouping model, user's request may be delayed up to the length of a period (D) even if there is no other request in the period for grouping. But, the period can be adjusted according to the arrival rate of user request. When the arrival rate of user request is high, grouping model can reduce the number of database access remarkably. 


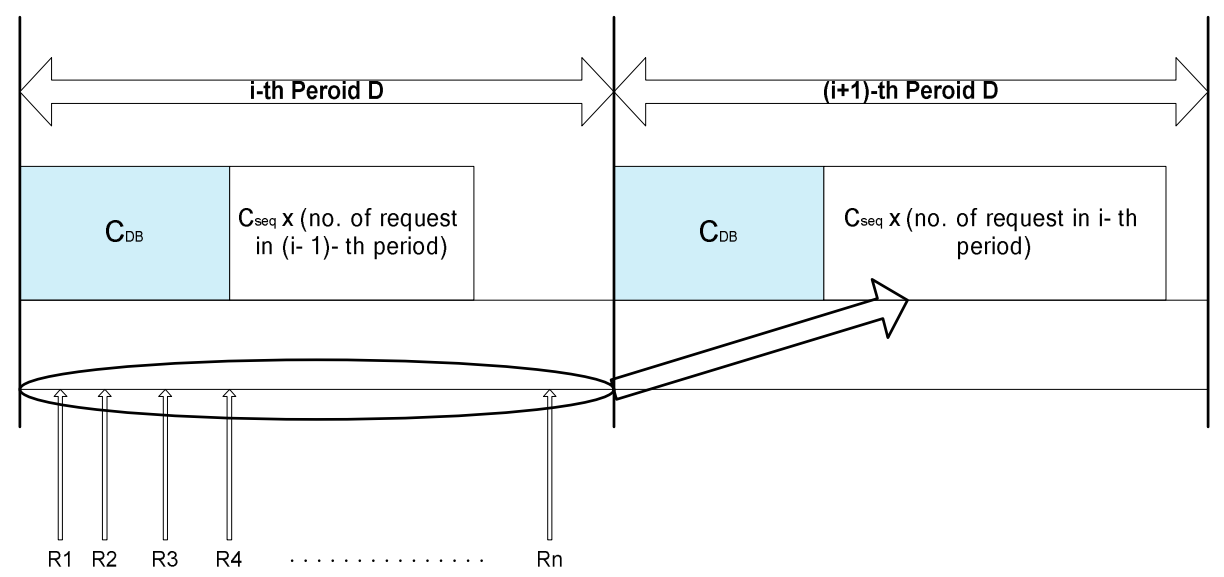

Fig. 2. Processing in grouping model

\subsection{Cost Analysis}

When the arrival of user request is governed by Poisson process with an arrival rate $\lambda$, the average number of user request arrived during a period (D) is $D \cdot \lambda$. Cost for comparison between gene sequences gathered from users' requests in a period and gene sequences in a database is $D \cdot \lambda \cdot C_{s e q}$. Cost for retrieving whole records in a database is assumed to $C_{D B}$. Thus, total system cost per period is as follows:

$$
C_{\text {total }}^{\text {srp }}=C_{D B}+D \cdot \lambda \cdot C_{s e q}
$$

An average cost to service a request is as follows:

$$
C_{\text {arg }}^{\text {grp }}=\frac{C_{D B}}{D \cdot \lambda}+C_{s e q}
$$

The probability of the arrival of at least one user's request during a period (D) is $1-e^{-\lambda D}$. Because the database is accessed only when there is a request during a period (D), an average cost to service a user for grouping model is as follows:

$$
C_{\text {avg }}^{\text {grp }}=\frac{C_{D B}}{D \cdot \lambda} \cdot\left(1-e^{-\lambda D}\right)+C_{s e q}
$$

Also, the average response time is the sum of retrieving all records in database and comparing them with a user request, and an average waiting time $(D / 2)$ until the end of period. The average number of user requests arrived during a period is $D \cdot \lambda$. Thus, time for processing data is $D \cdot \lambda \cdot C_{s e q}$ in a period (D). Because database is 
accessed once in a period, database access time is $C_{D B}$. Therefore, the average response for grouping model $\left(w_{g r p}\right)$ is as follows:

$$
W_{g r p}=C_{D B}+D \cdot \lambda \cdot C_{s e q}+\frac{D}{2}
$$

\section{Carpool Model}

Because users' requests gathered in a current period are processed in the next period in the grouping model, response time can be long. Retrieving whole database records is general in gene sequence alignment. Also, the result of sequence alignment is not affected on the order of sequence analyzed. Thus, we propose a model to process each user's request immediately by combining with the previously arrived requests to share and reduce database accesses without waiting until the end of period unlike the grouping model.

\subsection{Carpool Architecture}

In a carpool model, user's request is processed immediately with other requests being processed. When a server retrieves one record (sequence) from a sequence database, new request is processed together with the previously arrived requests.

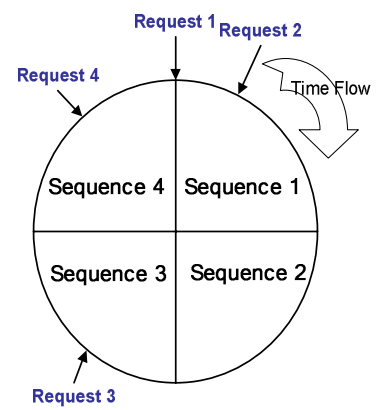

Fig. 3. Database access

Figure 3 shows an example. In the example, we assume a sequence database has four sequences (records), Sequence 1 to 4 . Sequence alignment program, such as FastA or Blast, compares the gene sequence from a request with all gene sequences in a database in the order of Sequence 1, 2, 3, and 4. Figure 3 shows that other users' requests, Request 2, Request 3, and Request 4, are arrived during the service for Request 1 . 

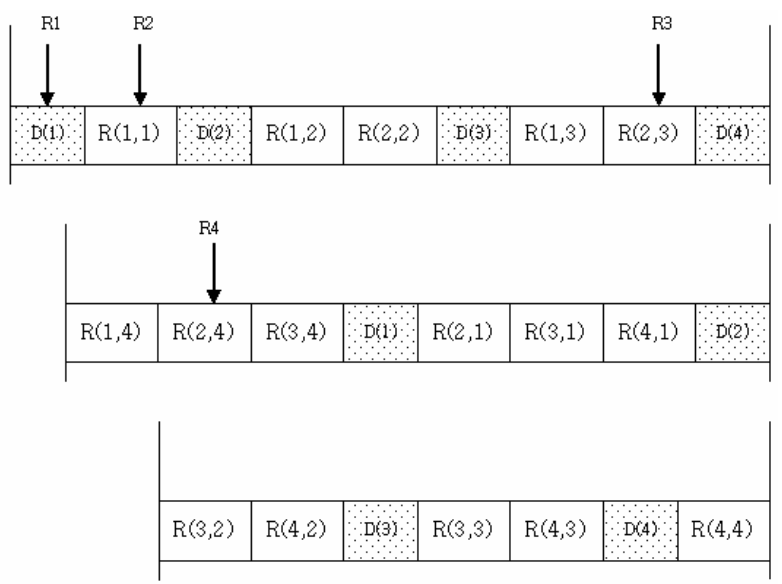

Fig. 4. Processing order in carpool mechanism

Figure 4 shows service procedures for the carpool model for four users' requests, Request 1 to 4. Database has four sequences, as shown in Fig.3. D(i) denotes a cost for retrieve the $i$-th sequence from a database, and $R(i, j)$ denotes comparing between Sequence $\mathrm{j}$ and a sequence in user Request $\mathrm{i}$.

Sequence 1 is retrieved $(\mathrm{D}(1))$ and compared with Request $1(\mathrm{R}(1,1))$. Because Request 2 is arrived while processing Request 1 , Sequence 2 is retrieved $(D(2))$ and compared with both requests, Request $1(\mathrm{R}(1,2))$ and Request $2(\mathrm{R} 2(2,2))$. Database access can be reduced because sequence is accessed once and Request 1 and Request 2 are processed together. Request 1 is finished after retrieve the Sequence 4 (D(4)) and process $(\mathrm{R}(1,4))$. After Sequence 4 is read $(\mathrm{D}(4))$ from a database and processed for three requests, Request $1(\mathrm{R}(1,4))$, Request $2(\mathrm{R}(2,4))$, Request $3(\mathrm{R}(3,4))$, Sequence 1 is retrieve $(D(1))$ to service Request 2, Request 3 and Request 4 because three requests have not been compared with Sequence 1. User's request of carpool model is processed immediately from the next retrieval of database record until all database records are retrieved and compared with the request sequence. Thus, in a carpool model, a new request does not have to wait unlike the grouping mechanism.

\subsection{Cost Analysis}

Total cost for sequence analysis adopting carpool model can be computed from the database retrieve time and processing time. The arrival rate of user's request is assumed to $\lambda$ governed by Poisson process. Time to retrieve all records in a database and process (compare) them with a sequence of user's request is assumed to $C_{\text {total }}^{c p}$. The average number of request is $\lambda \cdot C_{\text {total }}^{c p}$ during interval $C_{\text {total }}^{c p}$. All records in database is retrieved once $\left(C_{D B}\right)$ during the interval. Thus, total cost during the interval is computed as follows: ( $C_{s e q}$ is the same as the cost analysis in Sect. 2.2) 


$$
C_{\text {total }}^{c p}=C_{D B}+\lambda \cdot C_{\text {total }}^{c p} \cdot C_{\text {seq }}
$$

From formula (7), $C_{\text {total }}^{c p}$ can be computed as follows:

$$
C_{\text {total }}^{c p}=\frac{C_{D B}}{1-\lambda \cdot C_{s e q}}
$$

The probability of the arrival of at least one user's request within interval $C_{\text {total }}^{c p}$ is $1-e^{-\lambda C_{\text {total }}^{c p}}=1-e^{-\lambda \frac{C_{D B}}{1-\lambda \cdot C_{\text {seq }}}}$. The average cost of a carpool model can be computed as follows: (We derive analysis similar to formula (5), however use interval $C_{\text {total }}^{c p}$ instead of period D.)

$$
C_{\text {total }}^{c p}=\frac{C_{D B}}{C_{\text {total }}^{c p} \cdot \lambda} \cdot\left(1-e^{-\lambda \cdot C_{\text {total }}^{c p}}\right)+C_{s e q}=\left(\frac{1}{\lambda}-C_{s e q}\right) \cdot\left(1-e^{-\lambda \cdot \frac{C_{D B}}{1-\lambda} C_{s e q}}\right)+C_{s e q}
$$

Response time $\left(w_{c p}\right)$ of carpool model is the same as the sum of time to process user's request $\left(C_{D B}+C_{s e q}\right)$ and time to process other users' requests $\left(\lambda \cdot w_{c p} \cdot C_{s e q}\right)$ arrived during the response time $\left(w_{c p}\right)$.

$$
w_{c p}=C_{D B}+C_{s e q}+\lambda \cdot w_{c p} \cdot C_{s e q}
$$

From Eq. (10), $w_{c p}$ can be computed as follows:

$$
w_{c p}=\frac{C_{D B}+C_{s e q}}{1-\lambda \cdot C_{s e q}}
$$

\section{Performance Evaluation}

For performance evaluation, $C_{D B}$ and $C_{s e q}$ is measured by executing a real sequence alignment program FastA(version 3) accessing Genbank(Release 72.02) database, and cytochrome as sequence of user request on Pentium 4 system $(1.6 \mathrm{GHz}, 256 \mathrm{Mbyte}$ Ram (pc2700)). $C_{D B}$ and $C_{s e q}$ is $3.99 \mathrm{sec}$. and $19.98 \mathrm{sec}$., respectively, from the experiment. Cytochrome is a gene acting in cellular redox of human protein. 


\subsection{Cost Analysis}

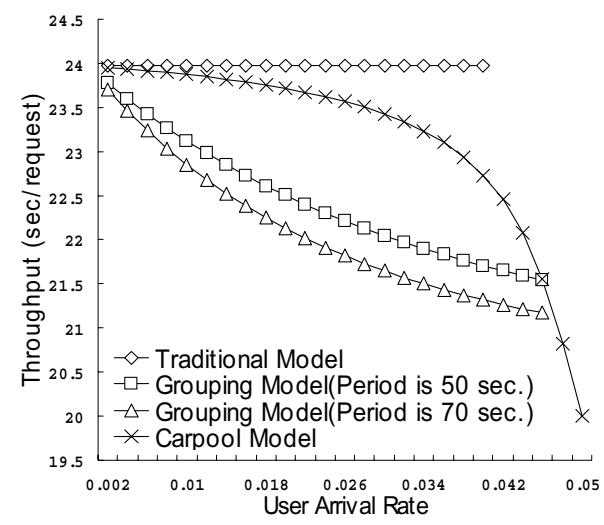

Fig. 5. Comparison of throughput (Average time per request)

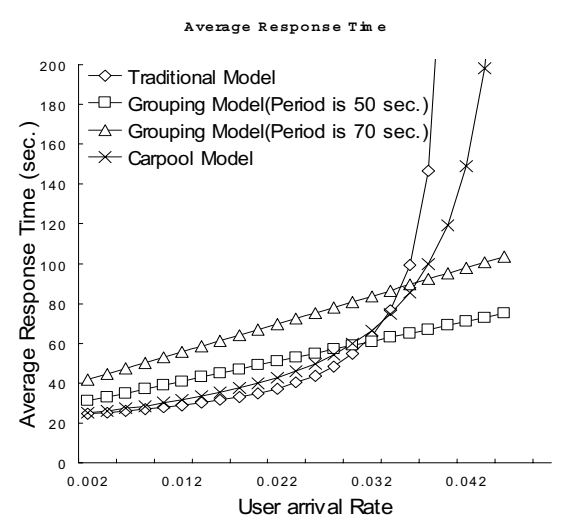

Fig. 6. Comparison of response time

Figure 5 depicts the average cost comparisons between traditional model, grouping model and carpool models. Period is $50 \mathrm{sec}$. and $70 \mathrm{sec}$. for grouping model. $\mathrm{X}$ axis is the rate of user request $(\lambda)$ and $y$ axis is system cost per user request. For grouping model and carpool model, system cost is decreased when $\lambda$ value increases by sharing a database retrieve. Also, for grouping model, system cost is reduced as D increases because more requests are sharing the database access. We draw curves up to the value of $\lambda$ where the system can admit the rate of arrival. Carpool model can admit the highest request rate (up to 0.05 ) while the traditional model accommodates the lowest (up to 0.04).

Figure 6 shows response time for different models. $X$ axis is request rate $(\lambda)$ and $y$ axis is an average response time for user's request. Because a request of traditional model is accumulated in a queue, response time is increased rapidly as request rate is increased. Response time of carpool model is shorter than that of grouping model at the low request rate because each request is immediately processed without grouping time unlike the grouping model.

\subsection{Simulation}

We simulated the three models to verify the analysis in Sects. 2.2, 3.2, and 4.2. $C_{D B}$ and $C_{s e q}$ are set to $3.99 \mathrm{sec}$. and $19.98 \mathrm{sec}$., respectively. We simulated grouping model with 2000 requests, and obtain an average response time. Also, carpool model is simulated with 60000 requests. The results of simulation are shown in Table 1. 
Table 1. Response time (seconds)

\begin{tabular}{|r||r|r|r|r|}
\hline $\begin{array}{c}\text { Request } \\
\text { rate }\end{array}$ & \multicolumn{1}{c|}{$\begin{array}{c}\text { tradition } \\
\text { model }\end{array}$} & $\begin{array}{c}\text { grouping } \\
(\mathrm{D}=50)\end{array}$ & $\begin{array}{c}\text { grouping } \\
(\mathrm{D}=70)\end{array}$ & $\begin{array}{c}\text { carpool } \\
\text { nodel }\end{array}$ \\
\hline \hline 0.020 & 35.45 & 61.62 & 81.08 & 39.98 \\
\hline 0.025 & 42.89 & 64.53 & 85.05 & 48.09 \\
\hline 0.030 & 57.07 & 68.98 & 90.99 & 60.35 \\
\hline 0.035 & 88.11 & 72.74 & 96.91 & 80.90 \\
\hline 0.040 & 254.20 & 76.85 & 106.71 & 121.33 \\
\hline 0.045 & 8067.45 & 83.66 & 122.56 & 244.82 \\
\hline 0.050 & 19153.61 & 91.18 & 143.89 & 7656.72 \\
\hline 0.055 & 28282.37 & 103.71 & 190.34 & 130092.20 \\
\hline
\end{tabular}

Figure 7 shows the graph from Table 1 . We obtain the very similar results comparing with the one obtained from the analysis (shown in Fig. 6).

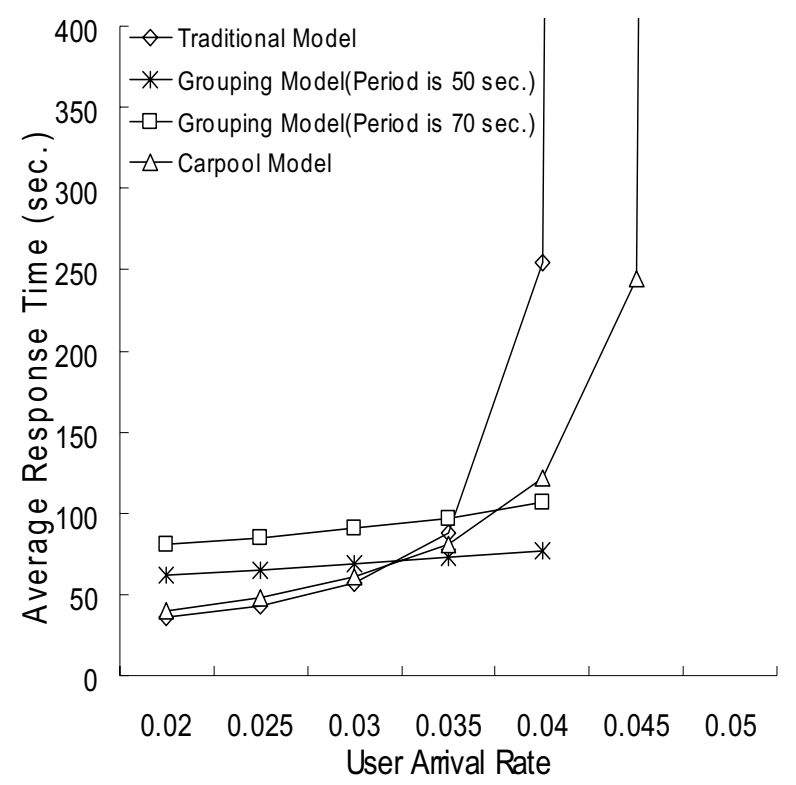

Fig. 7. Response time

We can know that response time of traditional model increases rapidly as the request rate increases. Grouping model shows the stable response time even if request rate increases. Carpool model has shorter response time than traditional model because each user's request is processed immediately and by sharing the database access. 


\section{Conclusion}

There are huge amount of data in gene or protein databases. To access such database efficiently, we propose two data sharing models, grouping and carpool models. Grouping model processes set of users' requests gathered in a period. By processing many users' requests together, database access can be shared, thus the number of database access can be reduced. Carpool model shares database access between requests and processes user's request immediately without waiting unlike the grouping model.

By these data sharing models, bioinformatics programs can retrieve database efficiently, reduce database access and admit higher rate of user request. Results from analysis and simulation show that these data sharing models can service more users as well as commit a shorter response time than a traditonal model.

\section{References}

[1] Ozden, B., R. Rastogi, and A. Silberschatz, and C. Martin "Demand Paging for Video on Demand Servers," IEEE International Conference on Multimedia Computing and Systems, May 1995.

[2] Stephen F. Altschul, Warren Gish, Webb Miller, Eugene W. Myers and David J. Lipman, "Basic Local Alignment Search Tool," J. Mol. Biol., pp. 403-410, 1990.

[3] Tieng K. Yap, Ophir Frieder, and Robert L. Martino, "Parallel Homologous Sequence Searching in Large Databases," IEEE Proceedings of the Fifth Symposium on the Frontiers of Massively Parallel Computation, 1995.

[4] Pearson, W.R., and Lipman, D. "Improved Tools for Biological Sequence Comparison," Proc. National Academic Science, USA, pp. 85, 1988.

[5] Jerry Banks, John S. Carson, II, Barry L. Nelson "Discrete-Event System Simulation," Prentice Hall, 2000.

[6] SWISSPROT: http: //www. expasy.org/

[7] GENBANK: http://www.ncbi.nlm.nih.gov/Genbank/

[8] EMBL: http://www.ncbi.nlm.nih.gov/Genbank/ 